\title{
Chia Seed Oil Ameliorates Doxorubicin-Induced Cardiotoxicity in Female Wistar Rats: An Electrocardiographic, Biochemical and Histopathological Approach
}

\author{
Akheruz Zaman Ahmed ${ }^{1} \cdot K_{\text {Kamalesh D. Mumbrekar }}^{2} \cdot$ Shakta Mani Satyam $^{3} \cdot$ Prakashchandra Shetty $^{1}$ (D) \\ Melanie Rose D'Souza ${ }^{1} \cdot$ Varun Kumar Singh ${ }^{4}$
}

Received: 30 November 2020 / Accepted: 2 March 2021 / Published online: 19 March 2021

(c) The Author(s) 2021

\begin{abstract}
Doxorubicin (DOX) is a potent anti-cancer antibiotic that was widely used for treatment of various cancers. It produces free radicals which result in extreme dose-limiting cardiotoxicity. This study investigated the cardioprotective potential of chia seed oil, an active polyphenolic nutraceutical against doxorubicin-induced cardiotoxicity in Wistar rats. Twenty-four female Wistar rats were divided into four groups $(n=6)$ which consist of normal control, DOX control, test-A and test-B group. Animals were prophylactically treated with two different doses of test drug, i.e. chia seed oil $2.5 \mathrm{ml} / \mathrm{kg} / \mathrm{day}$ and $5 \mathrm{ml} / \mathrm{kg} /$ day in test-A and test-B groups orally for 7 days. Doxorubicin $(25 \mathrm{mg} / \mathrm{kg}$; single dose) was administered intraperitoneally to DOX control, Test-A and Test-B animals on the seventh day to induce cardiotoxicity. ECG analysis was done before and after treatment. Besides ECG, CK, CK-MB, LDH, AST, MDA and GSH were analyzed. DOX had significantly altered ECG, CK, CK-MB, LDH, AST, MDA and GSH. Pre-treatment with chia seed oil significantly alleviated DOX-induced ECG changes and also guarded against DOX-induced rise of serum CK, CK-MB and AST levels. Chia seed oil alleviated histopathological alteration in DOX-treated rats. It also significantly inhibited DOX-induced GSH depletion and elevation of MDA. The present study revealed that chia seed oil exerts cardioprotection against doxorubicin-induced cardiotoxicity in female Wistar rats. Our study opens the perspective to clinical studies to precisely consider chia seed oil as a potential chemoprotectant nutraceutical in the combination chemotherapy with doxorubicin to limit its cardiotoxicity.
\end{abstract}

Keywords Anthracyclines · Chemotherapy · Acute cardiotoxicity · Electrocardiogram (ECG) · Nutraceuticals · Antioxidants

Handling Editor: Lorraine Chalifour.

Prakashchandra Shetty

prakashk.shetty@manipal.edu

1 Department of Anatomy, Melaka Manipal Medical College, Manipal Academy of Higher Education, Manipal, Karnataka, India

2 Department of Radiation Biology \&Toxicology, Manipal School of Life Sciences, Manipal Academy of Higher Education, Manipal, Karnataka, India

3 Department of Pharmacology, Melaka Manipal Medical College, Manipal Academy of Higher Education, Manipal, Karnataka, India

4 Department of Pathology, Melaka Manipal Medical College, Manipal Academy of Higher Education, Manipal, Karnataka, India

$\begin{array}{ll}\text { Abbreviations } \\ \text { DOX } & \text { Doxorubicin } \\ \text { CSO } & \text { Chia seed oil } \\ \text { CK-MB } & \text { Creatine kinase-MB } \\ \text { LDH } & \text { Lactate dehydrogenase } \\ \text { AST } & \text { Aspartate aminotransferase } \\ \text { CK } & \text { Creatine kinase } \\ \text { MDA } & \text { Malondialdehyde } \\ \text { GSH } & \text { Reduced glutathione } \\ \text { Nrf2 } & \text { Nuclear factor erythroid 2-related factor 2 } \\ \text { HO-1 } & \text { Heme oxygenase-1 } \\ \text { DMSO } & \text { Dimethyl sulfoxide } \\ \text { ROS } & \text { Reactive oxygen species } \\ \text { RNS } & \text { Reactive nitrogen species } \\ \text { H \& E } & \text { Haematoxylin and eosin } \\ \text { ECG } & \text { Electrocardiogram } \\ \text { TBA } & \text { Thiobarbituric acid } \\ \text { TCA } & \text { Trichloroacetic acid }\end{array}$




\section{DTNB 5, 5'-Dithiobis (2-nitrobenzoic acid) \\ PUFA Polyunsaturated fatty acids \\ CPCSEA Committee for the purpose of control and supervision on experiments on animals}

\section{Introduction}

Doxorubicin is a potent cytotoxic anticancer antibiotic separated from the culture of Streptomyces peucetius and used to treat different types of cancers like breast, ovarian, endometrial, bladder, thyroid, acute leukaemia, Hodgkin's disease, Wilms tumor, multiple myeloma [1]. Although it is a potent anticancer antibiotic, toxicities including nausea, vomiting, alopecia, and hematopoietic suppression, as well as a unique cardiotoxicity induced by DOX limit the use of this antibiotic. DOX is more prone to heart tissue toxicities and constitutes a major cause of morbidity and mortality in cancer patients [2].

An imbalance between the production of ROS and RNS free radicals and their elimination by the cellular antioxidant system results in oxidative stress. ROS and RNS are generated in mitochondria from dioxygen and nitric oxide, respectively. The heart is rich with mitochondria, which constitutes approximately $40 \%$ of the total intracellular volume of cardiomyocytes [3]. Since evidence points out to a preferential interaction of DOX with cardiac mitochondria, several mitochondrial alterations have been measured in different models [4]. DOX has great affinity for the negatively charged cardiolipin, which is abundant in the mitochondrial inner membrane [5] and leads to accumulation of DOX in this organelle. It was determined that under clinically relevant plasma DOX concentrations of $0.5-1 \mu \mathrm{M}$ can reach up to $50-100 \mu \mathrm{M}$ in mitochondria [6]. This high concentration of the drug leads to a high redox reactivity in the heart [7].

DOX-induced reactive oxygen species (ROS) causes oxidative damage to biological macromolecules, including lipids, proteins, DNA, and affects the structure and functions of cardiac cell membranes [8]. DOX also reduces endogenous antioxidants and increases lipid peroxidation that alters the cardiac function [9]. Besides, they significantly decrease the reduced glutathione (GSH) level. In general, antioxidant resources are low in cardiomyocytes as compared to other organs in the body, making the heart more vulnerable to free radical damage by DOX [10].

In cardiovascular research, electrocardiography (ECG) is widely used to monitor cardiac dysfunction because ECG is affordable and commonly available. The electrocardiographic changes associated with cardiomyopathy caused by doxorubicin initially include multiple reversible arrhythmias, most commonly sinus tachycardia [11, 12]. Some of the electrocardiographic features observed later with prolonged cardiotoxicity of doxorubicin are associated with T-wave flattening, QT-interval prolongation, and also $\mathrm{R}$ wave voltage loss [13].

Antioxidants are molecules that can suppress ROS and reduce oxidative stress damage. Even though extensive researches have been done to reduce cardiotoxicity caused by DOX, no effective preventive measure has yet been discovered. Chia seed oil (CSO) is derived from Chia seeds (Salvia hispanica L.) that are used traditionally for medicinal properties $[14,15]$. CSO contains high amount of polyunsaturated fatty acids (PUFA), mainly $\alpha$-linolenic acid (omega -3 fatty acids), oleic acids and palmitic acids [16]. Preclinical and clinical studies have verified that omega -3 fatty acids possess anti-inflammatory, antiarrhythmic, antithrombotic, neuroprotective, anticancer, antidepressant and immunomodulatory activities, making it an important class of drug and adjunctive therapeutics for disease therapy $[17,18]$.

The cardioprotective potential of chia seed oils has not been reported yet. Hence the present study aims to investigate the cardioprotective potential of chia seed oil against DOX-induced cardiotoxicity in female Wistar rats.

\section{Materials and Methods}

\section{Animals}

A total of 24 adult female Wistar rats (age: 8-10 weeks old \& body weight: 150-200 g) bred in Central Animal House, Manipal, Manipal Academy of Higher Education (MAHE) were housed in separate polypropylene cages. Animals were kept at temperatures $\left(22-24{ }^{\circ} \mathrm{C}\right), 12$-h light/12-h dark cycle and $40 \%-60 \%$ relative air humidity under standard conditions. Rats had continuous access to tap water with regular rat pellet diet on normal calories (Hindustan Lever Ltd., Mumbai, India). After randomization into various study groups, the rats were acclimatised for one week before starting the experiment under the same laboratory conditions. The experimental protocol was approved by the Institutional Animal Ethics Committee (IAEC/KMC/113/2019), and experiments were conducted in accordance with the ethical standards approved by the Ministry of Social Justice and Empowerment (Government of India) and the guidelines of CPCSEA.

\section{Chemicals}

Doxorubicin was purchased from Cipla Ltd., Goa (India). Assay kits for creatine kinase-MB (CK-MB), creatine kinase (CK), lactate dehydrogenase (LDH) and aminotransferase (AST) were obtained from ASPEN Laboratories, New 
Delhi (India). Thiobarbituric acid (TBA), trichloroacetic acid (TCA), 5,5'-dithiobis (2-nitrobenzoic acid) (DTNB) and reagents for histopathological analysis were procured from Sigma Aldrich-Merck, Bangalore (India). All reagents were analytical grade. Before the biochemical estimations, reagents were stabilized at room temperature for $30 \mathrm{~min}$.

\section{Extraction of Chia Seed Oil}

Extraction of oil from black Chia Seed was done at Bargi Naturals, Mysore, India using cold press extraction methods [19]. The oil was permitted to stand for a sedimentation time of 1 week after extraction to eliminate solid impurities. The oil was then filtered and placed in sealed dark coloured bottles protected by aluminium foils below $4^{\circ} \mathrm{C}$.

\section{Animal Selection and Grouping}

Baseline screening for any cardiac abnormality was done through BPL cardiart from the lead II ECG (BPL-91018, India) of all the experimental animals. Animals showing depressed ST segment/absence of P-wave/inverted P-wave/ non-specific ST segment/ST segment elevation were excluded from the experiment. A total of 24 rats showing normal ECG were included in the study and divided into four groups $(n=6)$ and treated as follows:

\section{Group I}

(Vehicle control): 2\% Dimethyl sulfoxide (DMSO) in double-distilled water; $1 \mathrm{ml} / \mathrm{kg} /$ day orally for 7 days $+0.9 \%$ $\mathrm{NaCl}, 1 \mathrm{ml} / \mathrm{kg}$ (single dose); i.p. on 7th day.

\section{Group II}

(DOX control): DOX $25 \mathrm{mg} / \mathrm{kg}$ (single dose); i.p. on 7th day.

\section{Group III}

(Test A; DOX + CSO $2.5 \mathrm{ml} / \mathrm{kg}$ ): CSO $2.5 \mathrm{ml} / \mathrm{kg} /$ day orally for 7 days + DOX $25 \mathrm{mg} / \mathrm{kg}$ (single dose); i.p. on 7 th day.

\section{Group IV}

(Test B; DOX + CSO $5 \mathrm{ml} / \mathrm{kg}$ ): CSO $5 \mathrm{ml} / \mathrm{kg} /$ day orally for 7 days + DOX $25 \mathrm{mg} / \mathrm{kg}$ (single dose); i.p. on 7th day.

On the 8th day ( $24 \mathrm{~h}$ after the administration of DOX), all the experimental animals were anaesthetized by intraperitoneal administration of both ketamine $(60 \mathrm{mg} / \mathrm{kg})$ and xylazine $(10 \mathrm{mg} / \mathrm{kg})$. The widely used therapeutic dose of doxorubicin is $60-75 \mathrm{mg} / \mathrm{m} 2 \mathrm{IV}$ once every 21 days to treat varieties of cancers. This dose is equivalent to $20-25 \mathrm{mg} / \mathrm{kg}$ in rats [20]. Two doses of chia seed oil $(2.5 \mathrm{ml} / \mathrm{kg}$ and $5 \mathrm{ml} /$ $\mathrm{kg}$ ) were selected based on our results of the acute toxicity study (ATC method; OECD 423 guideline) for chia seed oil.

\section{ECG Recording}

Following anaesthesia, each rat was positioned on the animal operation table for ECG recording. Electrodes were tied on palmer surface of clean-shaven limbs of the rat. The front limbs and left hind limb were used for the recording of ECG in standard leads, while the right hind limb was attached with grounded electrode. A conductive ECG gel was applied with care over each electrode to prevent a gel bridge between them from being formed. ECG was recorded for each animal for one minute and averages of data from 11 consecutive ECG signals were analysed quantitatively in terms of PR interval, QT interval, QTc interval and QRS complex amplitude. In addition to these ST segment was also analysed qualitatively.

\section{Collection of Blood and Serum Preparation}

Anaesthetized animals were euthanized after the blood collection. The heart was collected from the mediastinum by dissecting it out from the major blood vessels. Gross examination of the heart was done to check MI as per the criteria given by $\mathrm{Wu}$ et al. [21]. The heart was then washed in regular saline, soaked on blotting paper to extract the blood and then set for histopathological analysis in $10 \%$ formalin.

\section{Estimation of LDH, CK, CK-MB and AST}

LDH, CK, CK-MB and AST were measured using a semiautoanalyzer (Star 21 Plus, Mumbai, India) as per the standard protocol given along with the respective commercially available kits.

\section{Estimation of Malondialdehyde (MDA) \& Reduced Glutathione (GSH)}

Serum was analyzed for both MDA and GSH as per the protocol given by Satyam et al. [22]. Optical density was read at $540 \mathrm{~nm}$ and $412 \mathrm{~nm}$ for MDA and GSH, respectively, using iMark microplate absorbance reader (Bio-Rad Laboratories, USA). Serum MDA and GSH levels were calculated based on their absorbance and both were expressed as $\mathrm{mM} / \mathrm{ml}$.

\section{Histopathological Analysis}

After $24 \mathrm{~h}$ of fixation of heart tissues in $10 \%$ formalin, tissue samples were dehydrated in ascending concentrations of ethanol, cleared in xylene and embedded in paraffin to 
prepare the block. Then, histological sections of $5 \mu \mathrm{m}$ thicknesses were taken using rotary microtome and staining was done using Haematoxylin \& Eosin (H \& E). Inter muscular edema, myofibrillar loss, infiltration with inflammatory cells, vacuolization \& cardiomyocytes degeneration were assessed from all the experimental groups. All the pathological findings were verified by a pathologist.

\section{Statistical Analysis}

Using the Statistical Package for the Social Sciences (SPSS version 16.0; SPSS), data expressed as mean \pm standard deviation and analyzed by one-way analysis of variance (ANOVA) followed by post hoc Tukey test. A level for $p \leq 0.05$ was considered to be statistically significant.

\section{Results}

\section{Effect on Electrocardiography}

QT interval prolongation, reduction in QRS complex and ST segment changes are some of the characteristic ECG findings in DOX-induced cardiomyopathy. In DOX-treated animals, increased PR interval, prolongation of QT \& QTc interval, reduced QRS complex amplitude and non-specific
ST segment were observed. However, these changes were not seen in vehicle control and test groups (Fig. 1).

There was a significant increase in $\mathrm{PR}$ interval $(\mathrm{p}=0.003), \mathrm{QT}$ interval $(p<0.001)$, QTc interval $(p<0.001)$ and decrease in QRS complex amplitude $(p<0.001)$ among DOX control animals in comparison with normal control animals. PR interval $(p<0.001)$, QT interval $(p=0.003)$, QTc interval $(p=0.003)$ were significantly decreased and QRS complex amplitude $(p=0.010)$ was increased among Test-A (DOX + CSO $2.5 \mathrm{ml} / \mathrm{kg}$ ) treated group compared to DOX control group. A significant decrease was also observed in PR interval $(p<0.001)$, QT interval $(p<0.001)$, QTc interval $(p<0.001)$ and increase in QRS complex amplitude $(p<0.001)$ in Test-B (DOX + CSO $5 \mathrm{ml} / \mathrm{kg}$ ) group in comparison with DOX control animals (Fig. 2).

\section{Amelioration of Serum CK-MB, CK, LDH and AST Activity}

Measurements of the serum cardiac markers like CK-MB, CK, LDH and AST were done to understand cardiotoxicity induced by DOX and the protective effect of CSO. There was a significant increase in CK-MB $(p<0.01)$, CK $(p<0.001)$, LDH $(p<0.001)$ and AST $(p<0.001)$ in DOX control group in comparison with normal control. A significant decrease was observed for CK-MB $(p<0.001)$

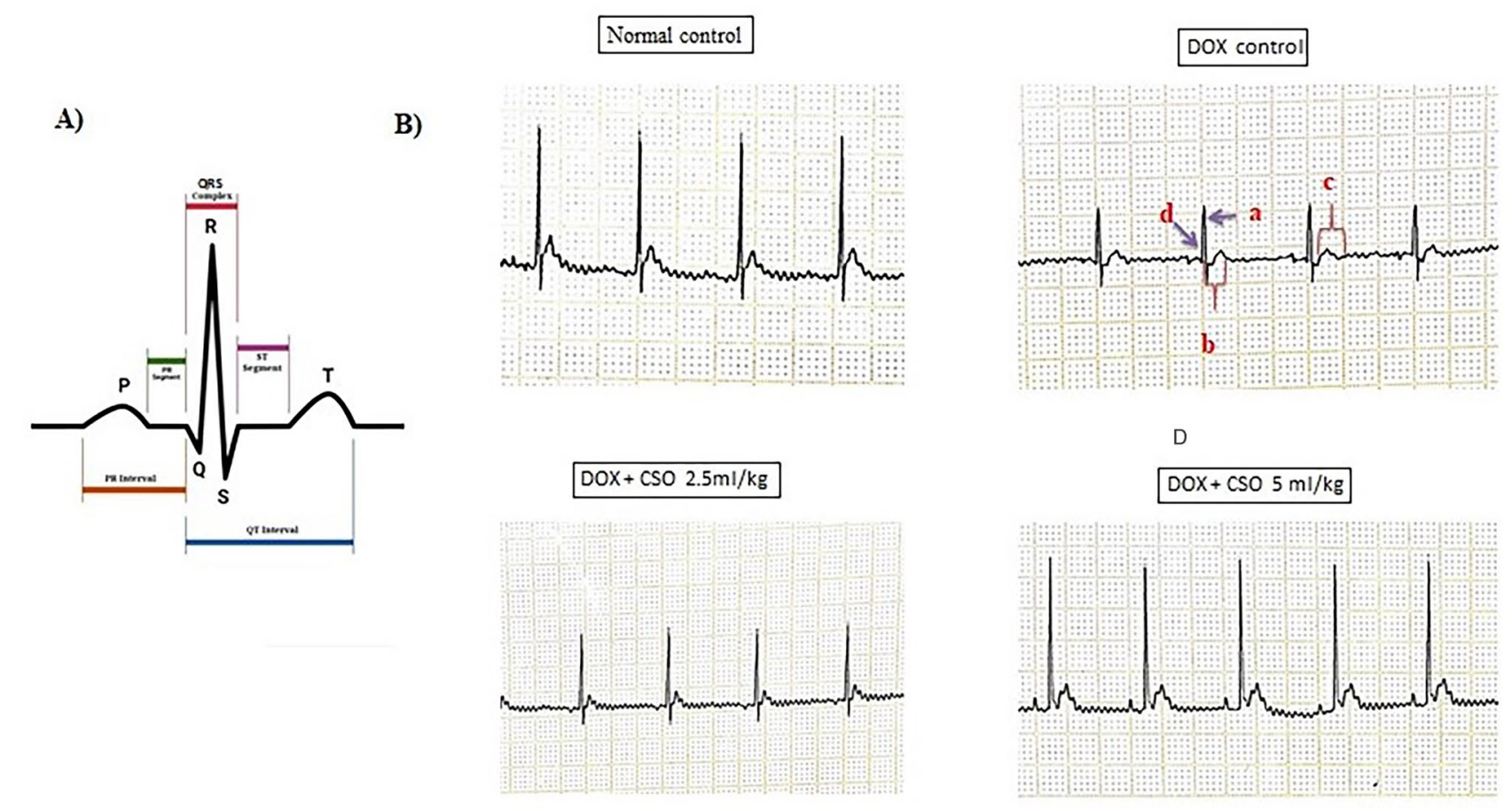

Fig. 1 A Typical ECG graph showing PR interval, QRS complex, ST segment, QT interval. B Representative ECG of normal control, DOX control, DOX + CSO $2.5 \mathrm{ml} / \mathrm{kg}$ and DOX + CSO $5 \mathrm{ml} / \mathrm{kg}$. a Reduced
QRS complex amplitude, b Prolongation of QT interval, c Non-specific ST segment, $\mathbf{d}$ Increased PR interval 


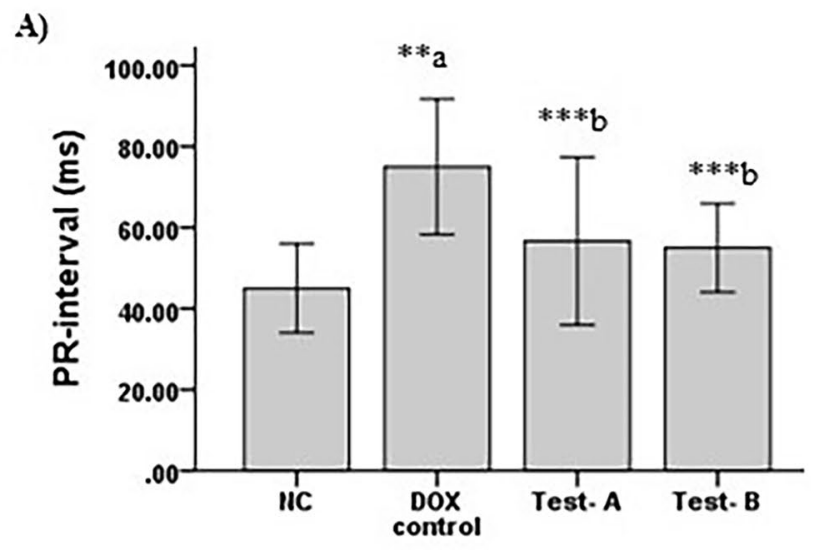

C)

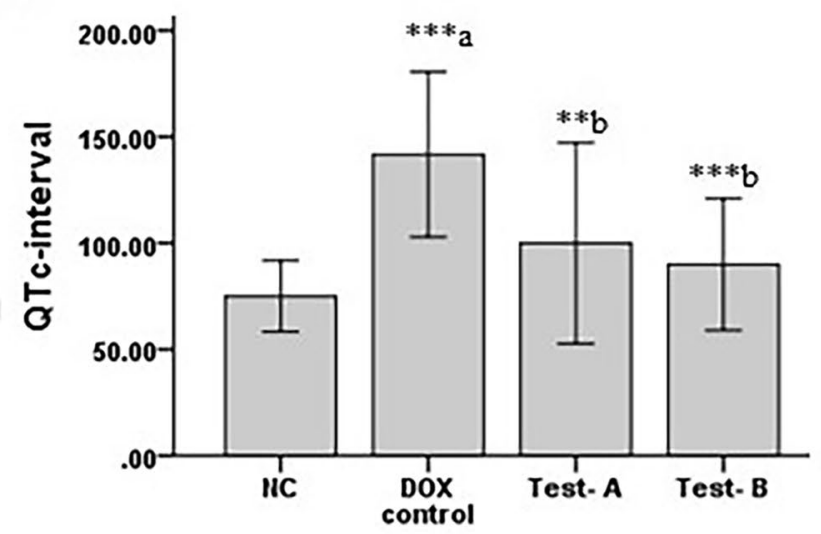

Fig. 2 Effect of DOX and CSO on ECG parameters. A PR interval (ms), B QT interval (ms), C QTc-interval (ms), D QRS complex (ms). Data were represented as mean $\pm \mathrm{SD}$, and one-way ANOVA fol-

and CK $(p<0.001)$ in the Test-A group. Similarly in Test-B group also a decrease in CK-MB $(p<0.001)$, CK $(p<0.001)$ and AST $(p<0.01)$ was observed as compared to DOX control group. CSO $5 \mathrm{ml} / \mathrm{kg}$ has significantly reduced CK-MB $(p<0.001)$ compared to CSO $2.5 \mathrm{ml} / \mathrm{kg}$ treated group (Fig. 3). The increase in CK-MB, CK, LDH and AST indicates cellular damage and loss of functional integrity of cardiomyocytes. These findings had shown cardiotoxicity by DOX in female Wistar rats. Treatment with CSO ameliorated increase AQ levels of CK-MB, CK and AST, indicating its cardioprotective effect against DOX.

\section{Effect on Serum GSH and MDA Levels}

In the DOX control group, there was a significant decrease in GSH $(p<0.001)$ whereas MDA $(p=0.015)$ was significantly increased compared to normal control animals. CSO $2.5 \mathrm{ml} / \mathrm{kg}$ (Test-A) has significantly increased GSH $(p<0.001)$ and decreased MDA $(p<0.001)$ levels in
B)

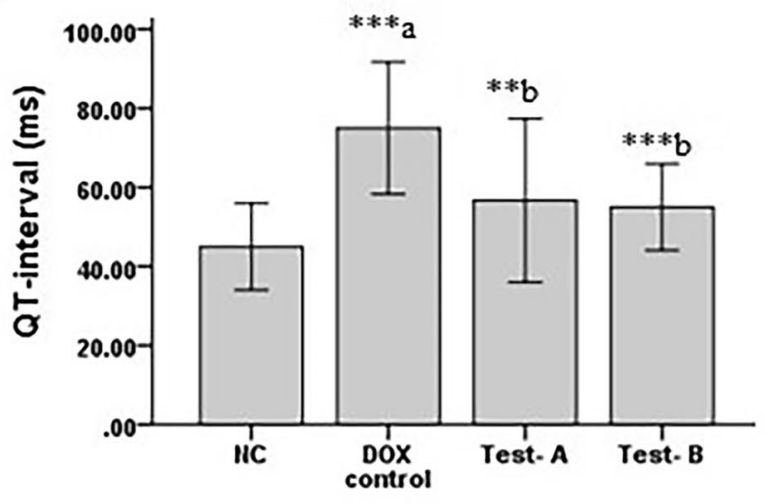

D)

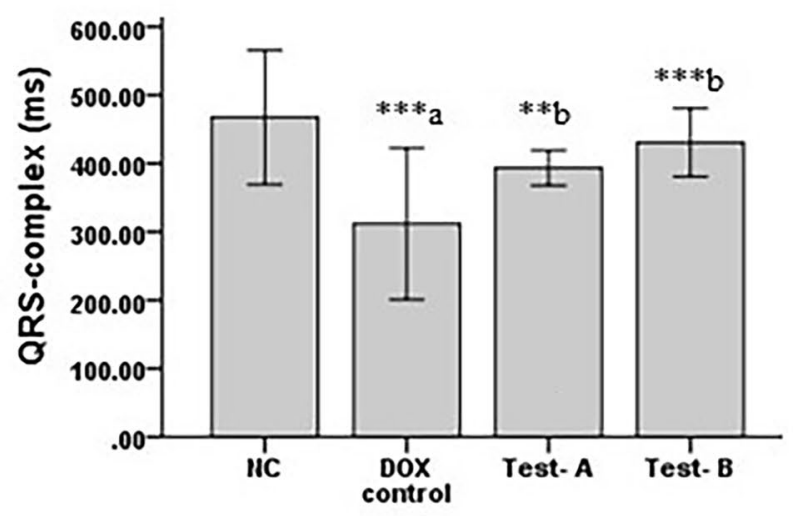

lowed by Tukey's post hoc test $(* * * p \leq 0.001, * * p \leq 0.01, * p \leq 0.05$ a compared to control, ${ }^{\mathrm{b}}$ compared to DOX control), NC: Normal control, Test-A: DOX + CSO $2.5 \mathrm{ml} / \mathrm{kg}$ and Test-B: DOX + CSO $5 \mathrm{ml} / \mathrm{kg}$

comparison with the rats treated with the DOX control group. Similarly, there was a significant increase in GSH $(p=0.004)$ and decrease in MDA $(p<0.001)$ among the rats treated with CSO $5 \mathrm{ml} / \mathrm{kg}$ compared to the DOX control group (Fig. 4).

\section{Gross Examination of Isolated Hearts}

Myocardial infarction (MI) was commonly noted among isolated hearts of DOX control rats. MI as pale/yellow with hyperemic or hemorrhagic borders/white-grey (scar) was significantly noted among isolated hearts of DOX control rats, whereas these were absent in normal control and test groups (Fig. 5a).

\section{Histopathological Examination of Cardiac Tissue}

DOX-induced cardiomyopathy was further examined by using H\&E staining under a light microscope. The normal control group had shown typical cardiomyocytes 
A)

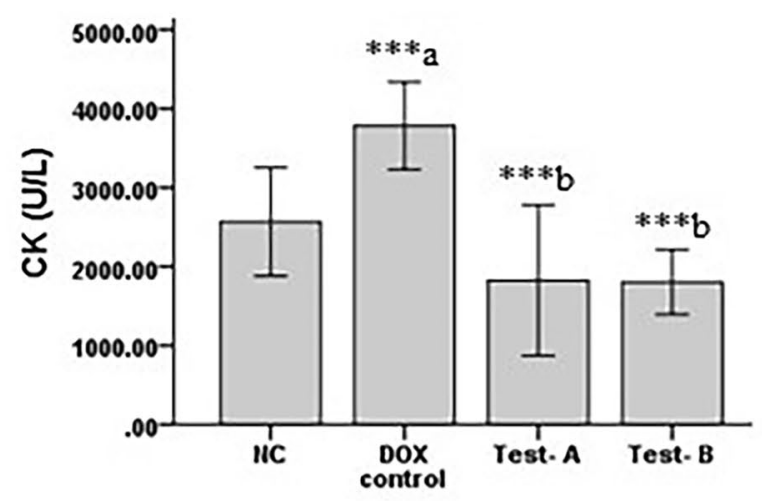

C)

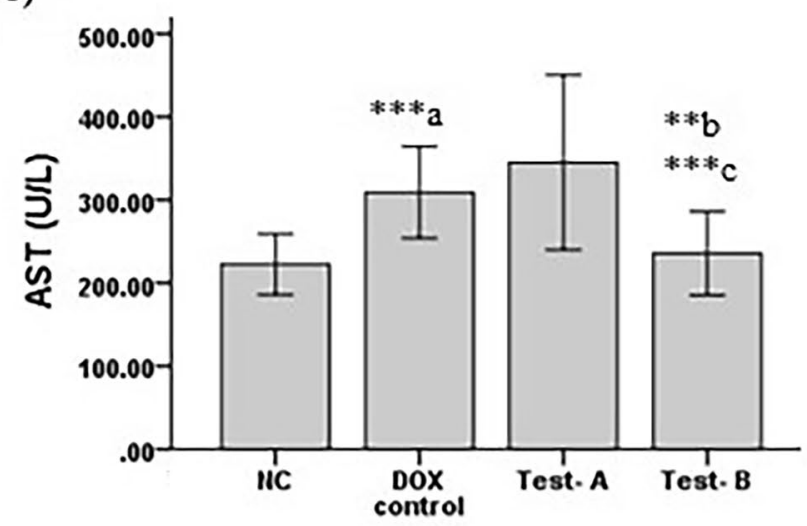

Fig. 3 Effect of DOX and CSO on serum cardiac injury biomarkers. A Creatinine kinase. B Creatinine kinase-MB. C Aspartate aminotransferase. D Lactate dehydrogenase. Data were represented as mean $\pm \mathrm{SD}$, one-way ANOVA followed by Tukey's post hoc test

A)

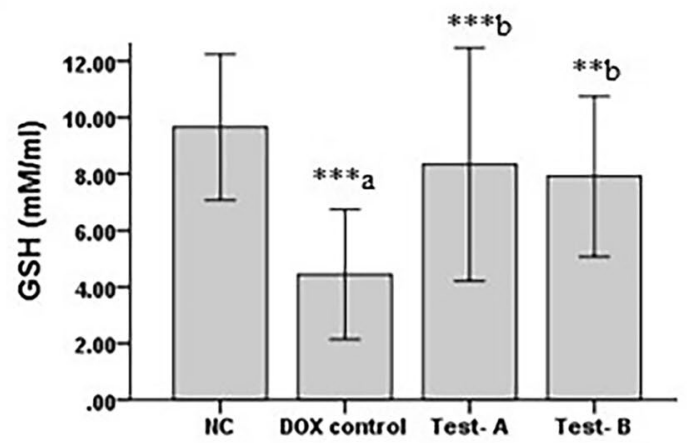

Fig. 4 Effect of DOX and CSO on Oxidative stress parameters. A Reduced glutathione. B Malondialdehyde.. Data were represented as mean $\pm \mathrm{SD}$, one-way ANOVA followed by Tukey's post hoc test

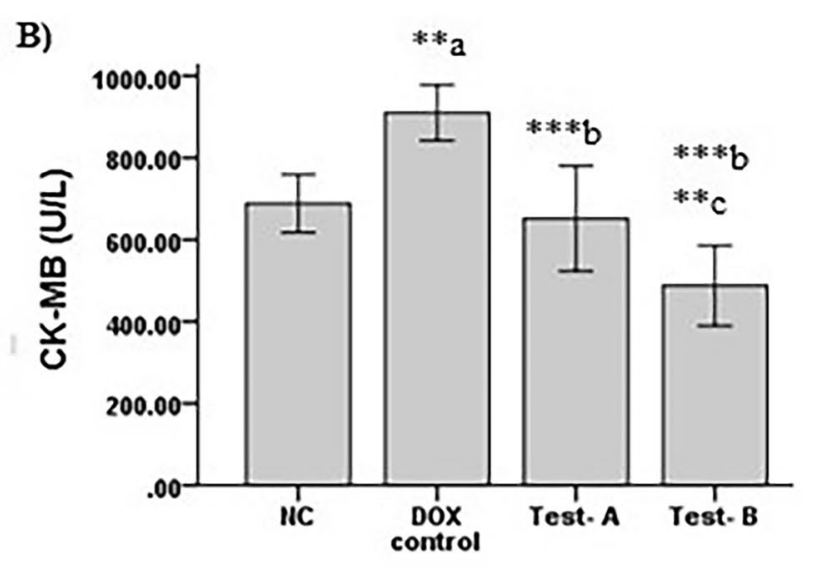

D)

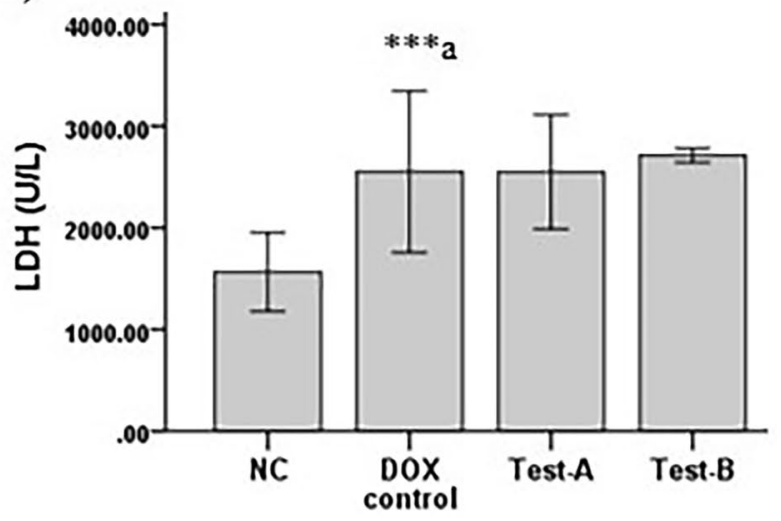

$\left(* * * p \leq 0.001, * * p \leq 0.01, * p \leq 0.05{ }^{\text {a }}\right.$ compared to control, ${ }^{\mathrm{b}}$ compared to DOX control), NC: Normal control, Test-A: DOX + CSO $2.5 \mathrm{ml} / \mathrm{kg}$ and Test-B: DOX + CSO $5 \mathrm{ml} / \mathrm{kg}$

B)

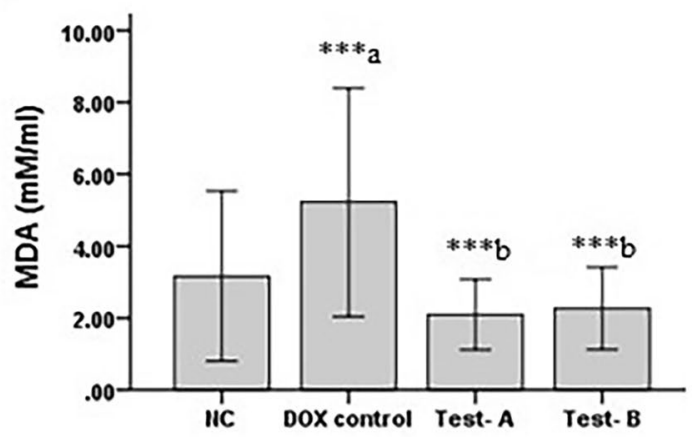

$\left({ }^{* * *} p \leq 0.001,{ }^{* *} p \leq 0.01,{ }^{*} p \leq 0.05^{\mathrm{a}}\right.$ compared to control, ${ }^{\mathrm{b}}$ compared to DOX control), NC: Normal control, Test-A: DOX +CSO $2.5 \mathrm{ml} / \mathrm{kg}$ and Test-B: DOX + CSO $5 \mathrm{ml} / \mathrm{kg}$ 
A)
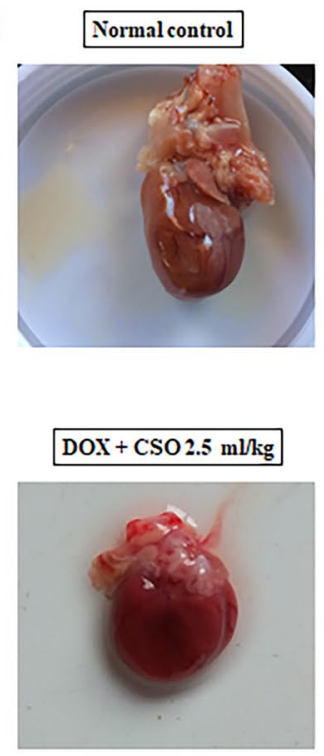
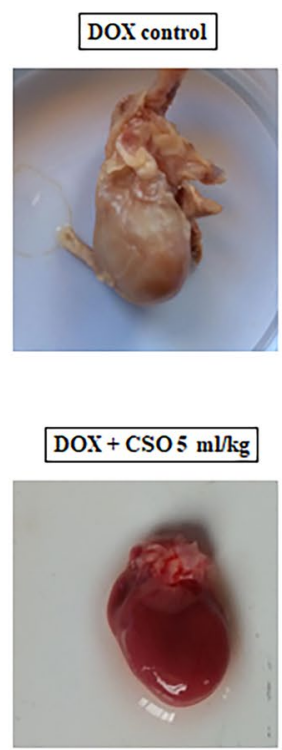

B)

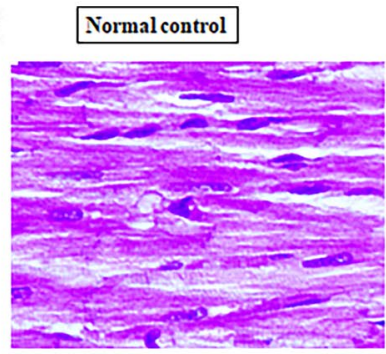

$\mathrm{DOX}+\mathrm{CSO} 2.5 \mathrm{~m} / \mathrm{kg}$

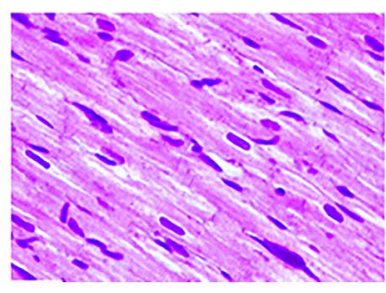

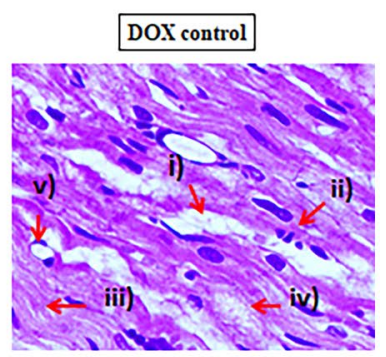

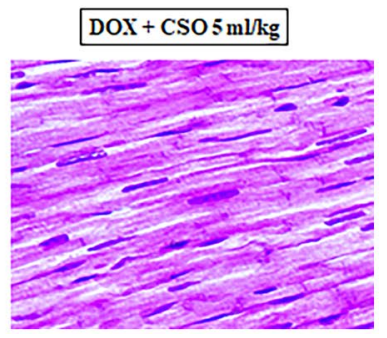

Fig. 5 Effect of DOX and CSO on heart anatomy. A Representative photographs of isolated hearts after the end of the experiment. B Longitudinal section of cardiac tissue stained with $\mathrm{H} \& \mathrm{E}$ under 400X,

architecture. In DOX-treated control group's cardiomyocytes had presented intermuscular edema, myofibrillar loss, infiltration with inflammatory cells, vacuolization and cardiomyocytes degeneration. All these pathological changes were mitigated among test-A and test-B group and cardiomyocytes architecture almost looked like normal control group (Fig. 5b).

\section{Discussion}

DOX-induced cardiotoxicity is known to have an extremely severe adverse effect of oncology therapy. After its discovery, multiple molecular mechanisms have been suggested to explain the pathogenesis of acute and chronic cardiotoxicity caused by DOX, including oxidative stress, iron metabolism, $\mathrm{Ca} 2+$ homeostasis dysregulation, gene expression modulation, sarcomeric structure alterations and apoptosis [23]. Different methods to protect the heart during cancer treatment have been developed based on these mechanisms. Chia seed is an annual herbaceous plant and rich in antioxidants. This study evaluated the cardioprotective potential of chia seed oil against DOX-induced cardiotoxicity in female Wistar rats. The results of this study showed cardioprotective potential of chia seed oil in female Wistar rats against DOX-induced cardiotoxicity. DOX is more commonly used in the treatment of breast cancer. Hence female Wistar rats were selected in this study.

Acute cardiotoxicity caused by DOX is observed during and within 2-3 days of its single dose of DOX administration

( i Inter muscular edema, ii Infiltration with inflammatory cells, iii Myofibrillar loss, iv Cardiomyocytes degeneration, v Vacuolization)

$[24,25]$. The present study has observed acute cardiotoxicity with a single dose of DOX administration to the experimental animals. DOX has significantly altered ECG waves in the form of increased PR interval, prolonged QT and QTc interval, reduced QRS complex amplitude and nonspecific ST segment. The findings of all these ECG changes in the present study were supported by previously reported studies [26-28]. Due to DOX-induced lipid peroxidation, an altered membrane structure may be responsible for most ECG changes. Both the doses of chia seed oils $(2.5 \mathrm{ml} / \mathrm{kg}$ and $5 \mathrm{ml} / \mathrm{kg}$ ) have significantly mitigated these acute changes in ECG.

The present study had observed significantly high serum LDH, CK, CK-MB and AST in DOX control group as compared to normal control group. Previously, studies had reported high serum LDH, CK, CK-MB and AST levels in rats treated with DOX that is corroborated with the finding of the present study:, serum cardiac biomarkers are released from damaged cardiomyocytes and are sensitive indicators of DOX-induced cardiotoxicity [29, 30]. CSO had significantly inhibited DOX-induced elevation of serum CK, CK-MB and AST levels. These biochemical data and ECG abnormalities were further supported by histopathological examination of cardiomyocytes. The histopathological examination of cardiac tissue had shown cardiac injury in the form of intermuscular edema, myofibrillar loss, infiltration with inflammatory cells, vacuolization and cardiomyocytes degeneration. These histological findings are linked with the previously published data $[19,30]$. Pre-treatment with CSO had reduced DOX-induced histological alteration 
of cardiomyocytes as compared to those only treated with DOX. The observed protection of cardiomyocytes integrity might lead to decreased leakage of cardiac bio-markers in serum that was observed in this study.

Oxidative stress is a keystone in DOX-induced cardiotoxicity [31], hence this present study also investigated the non-enzymatic antioxidant status of the animals. The data had shown significantly high MDA in DOX control group as compared to normal control group and GSH was significantly reduced following DOX administration. These data clearly show that there is overt oxidative stress. The findings of the present study data are linked with previous investigations $[32,33]$. The deficiency of GSH and the increase in MDA level produced by DOX may be attributed to the depletion of GSH in the interactions of DOX-induced free radicals with biomembrane and following lipid peroxidation. Pre-treatment with CSO significantly protects the animals against the DOX-induced oxidative stress. Our results had shown that CSO improved the antioxidant activities in rats that were decreased by DOX. This might be on the basis of its high content of numerous antioxidants including $\alpha$-linolenic acid and polyphenols [16]. Because of its potent anti-oxidant properties, CSO were reported to inhibit oxidative induced damages $[34,35]$. Many studies have reported that $\alpha$-linolenic acid possess potent anti-inflammatory, antioxidant, antiarrhythmic, antithrombotic, neuroprotective, anticancer, antidepressant and immunomodulatory properties $[17,18]$.

ROS is a small biological molecule that is continuously synthesized as a natural end product of oxygen metabolism in aerobic organisms. In cell signalling, ROS plays a significant physiological function, while long-term cell exposure to elevated ROS levels will produce profound toxic effects, such as apoptotic cell death and necrotic [36]. Non-enzymatic synthesis of ROS occurs primarily in mitochondria, especially in the mitochondrial electron transport chain complexes I and III [37]. Oxidative stress is defined by the deleterious processes induced by an imbalance between the formation of ROS and minimal antioxidant defences. Disturbance of the cellular redox balance, due to increased ROS synthesis and limited antioxidant defences, contributes to oxidative changes of biological macromolecules such as nucleic acids, lipids and proteins [38]. Particularly, mitochondrial DNA is highly vulnerable to ROS-induced damages because it is located in close proximity to the synthesis site of ROS and mitochondrial DNA repair mechanisms are hampered. As a consequence, mitochondrial DNA deletions accumulate, eventually leading to a decrease in mitochondrial function and concomitant increased production of ROS and cell becomes more prone to death [39].

ROS is produced by one of the mechanisms of DOX to induce toxicity [20]. One of the studies reported that cardiomyocytes contain highest number of mitochondria which are the main source for ROS production [3]. Antioxidants are the keys of defence mechanisms to scavenge ROS to protect the host against damages [40]. With its rich antioxidant properties, $\mathrm{CHO}$ has the ability to scavenge ROS produced by DOX and protect the tissue from oxidative stress damages. Many studies reported that pre-treatment with omega-3 fatty acids as a dominant constituent of chia seed oils reduces the detrimental oxidative effects of myocardial infarction because of its potent antioxidant properties either by increasing the activity of antioxidant enzymes such as catalase, superoxide dismutase or by increasing the level of non-enzymatic antioxidant markers such as GSH [41].

The elevated ROS level also decreases the expression of the nuclear factor erythroid 2-related factor (Nrf2), which enhances cell susceptibility to more oxidative stress and apoptosis [42]. DOX has been reported to decrease Nrf2/ HO-1 expression to induce oxidative stress-mediated injury [43]. Many studies have reported that chia seed oil modulates Nrf2/HO-1 pathway and thereby protects from DOX-induced oxidative injury [44-46]. In this study, the inhibitory effect of chia seed oil on oxidative stress was demonstrated by a decrease in MDA and an increase in GSH levels, which indicates its anticipated cardioprotective mechanism. The normalisation of serum CK, CK-MB and AST activities supports the defence of chia seed oil against heart injury through a potential membrane stabilising effect.

\section{Conclusion}

The present study collectively demonstrated that chia seed oil could protect against DOX-induced cardiac toxicity in female Wistar rats by inhibiting oxidative stress. Our study opens the perspective to clinical studies to precisely considering chia seed oil as a potential chemoprotectant nutraceutical in the combination chemotherapy with DOX to limit its cardiotoxicity.

Acknowledgements The authors acknowledge the support of Manipal Academy of Higher Education, Manipal, Karnataka, India for allowing all the lab facilities to carry out all the experiments. The study was financially supported by Intramural fund given by Manipal Academy of Higher Education for which the authors are grateful.

Funding Open access funding provided by Manipal Academy of Higher Education, Manipal. This work was financially supported by Manipal Academy of Higher Education, Manipal (India).

Data Availability All data are available and can be given as per request.

Code Availability Not applicable. 


\section{Declarations}

Conflict of interest The authors have no conflicts of interest that are directly relevant to the content of this manuscript.

Ethical Approval The experimental protocol was approved by the Institutional Animal Ethics Committee (IAEC/KMC/113/2019).

\section{Consent to Participate Not applicable.}

Consent for Publication All the authors are ready to publish the manuscript in 'Cardiovascular Toxicology' as per rule and regulations of the journal.

Open Access This article is licensed under a Creative Commons Attribution 4.0 International License, which permits use, sharing, adaptation, distribution and reproduction in any medium or format, as long as you give appropriate credit to the original author(s) and the source, provide a link to the Creative Commons licence, and indicate if changes were made. The images or other third party material in this article are included in the article's Creative Commons licence, unless indicated otherwise in a credit line to the material. If material is not included in the article's Creative Commons licence and your intended use is not permitted by statutory regulation or exceeds the permitted use, you will need to obtain permission directly from the copyright holder. To view a copy of this licence, visit http://creativecommons.org/licenses/by/4.0/.

\section{References}

1. Young, R. C., Ozols, R. F., \& Myers, C. E. (1981). The anthracycline antineoplastic drugs. New England Journal of Medicine, 305(3), 139-153.

2. Outomuro, D., Grana, D. R., Azzato, F., \& Milei, J. (2007). Adriamycin-induced myocardial toxicity: new solutions for an old problem. International journal of cardiology, 117(1), 6-15.

3. Goffart, S., von Kleist-Retzow, J. C., \& Wiesner, R. J. (2004). Regulation of mitochondrial proliferation in the heart: power-plant failure contributes to cardiac failure in hypertrophy. Cardiovascular research, 64(2), 198-207.

4. Carvalho, F. S., Burgeiro, A., Garcia, R., Moreno, A. J., Carvalho, R. A., \& Oliveira, P. J. (2014). Doxorubicin-induced cardiotoxicity: from bioenergetic failure and cell death to cardiomyopathy. Medicinal research reviews, 34(1), 106-135.

5. Parker, M. A., King, V., \& Howard, K. P. (2001). Nuclear magnetic resonance study of doxorubicin binding to cardiolipin containing magnetically oriented phospholipid bilayers. Biochimica et Biophysica Acta (BBA)-Biomembranes, 1514(2), 206-216.

6. Sarvazyan, N. A. (1996). Visualization of doxorubicin-induced oxidative stress in isolated cardiac myocytes. American Journal of Physiology-Heart and Circulatory Physiology, 271(5), H2079-H2085.

7. Alexieva, B., Sainova, I., Pavlova, V., Markova, T., Valkova, I., \& Nikolova, E. (2014). Insights into mechanisms of doxorubicin cardiotoxicity. J Phys Pharm Adv, 4(3), 342-348.

8. Kuznetsov, A. V., Margreiter, R., Amberger, A., Saks, V., \& Grimm, M. (2011). Changes in mitochondrial redox state, membrane potential and calcium precede mitochondrial dysfunction in doxorubicin-induced cell death. Biochimica et Biophysica Acta (BBA)-Molecular Cell Research, 1813(6), 1144-1152.

9. Singal, P., Li, T., Kumar, D., Danelisen, I., \& Iliskovic, N. (2000). Adriamycin-induced heart failure: mechanisms and modulation. Molecular and cellular biochemistry, 207(1-2), 77-86.
10. Halestrap, A. P. (2006). Calcium, mitochondria and reperfusion injury: a pore way to die. Biochemical Society Transactions, 34, 232-237.

11. Cardinale, D., Colombo, A., Bacchiani, G., Tedeschi, I., Meroni, C. A., Veglia, F., et al. (2015). Early detection of anthracycline cardiotoxicity and improvement with heart failure therapy. $\mathrm{Circu}$ lation, 131(22), 1981-1988.

12. Kim, H., Chung, W. B., Cho, K. I., Kim, B. J., Seo, J. S., Park, S. M., et al. (2018). Diagnosis, treatment, and prevention of cardiovascular toxicity related to anti-cancer treatment in clinical practice: an opinion paper from the working group on CardioOncology of the Korean Society of Echocardiography. Journal of cardiovascular ultrasound, 26(1), 1-25.

13. Gilladoga, A. C., Manuel, C., Tan, C. T., Wollner, N., Sternberg, S. S., \& Murphy, M. L. (1976). The cardiotoxicity of adriamycin and daunomycin in children. Cancer, 37(S2), 1070-1078.

14. Suri, S., Passi, S.J., \& Goyat, J., (2016). Chia seed (Salvia hispanica L.) - A new age functional food. In 4th International Conference on Recent Innovations in Science Engineering and Management, 286-299.

15. Munoz, L. A., Cobos, A., Diaz, O., \& Aguilera, J. M. (2013). Chia seed (Salvia hispanica): an ancient grain and a new functional food. Food reviews international, 29(4), 394-408.

16. Kulczyński, B., Kobus-Cisowska, J., Taczanowski, M., Kmiecik, D., \& Gramza-Michałowska, A. (2019). The chemical composition and nutritional value of chia seeds-Current state of knowledge. Nutrients, 11(6), 1242.

17. Simopoulos, A. P. (2002). Omega-3 fatty acids in inflammation and autoimmune diseases. Journal of the American College of nutrition, 21(6), 495-505.

18. Gil, A. (2002). Polyunsaturated fatty acids and inflammatory diseases. Biomedicine \& pharmacotherapy, 56(8), 388-396.

19. Ozcan, M. M., Al-Juhaimi, F. Y., Ahmed, I. A. M., Osman, M. A., \& Gassem, M. A. (2019). Effect of soxhlet and cold press extractions on the physico-chemical characteristics of roasted and non-roasted chia seed oils. Journal of Food Measurement and Characterization, 13(1), 648-655.

20. Hajra, S., Patra, A. R., Basu, A., \& Bhattacharya, S. (2018). Prevention of doxorubicin (DOX)-induced genotoxicity and cardiotoxicity: Effect of plant derived small molecule indole3 -carbinol (I3C) on oxidative stress and inflammation. Biomedicine \& Pharmacotherapy, 101, 228-243.

21. Wu, Y., Yin, X., Wijaya, C., Huang, M. H., \& McConnell, B. K. (2011). Acute myocardial infarction in rats. JoVE (Journal of Visualized Experiments), 48, e2464.

22. Satyam, S. M., Bairy, L. K., Pirasanthan, R., \& Vaishnav, R. L. (2014). Grape seed extract and zinc containing nutritional food supplement prevents onset and progression of age-related cataract in Wistar rats. The journal of nutrition, health \& aging, 18(5), 524-530.

23. Dos Santos, D. S., \& dos Santos Goldenberg, R. C. (2018). Doxorubicin-Induced Cardiotoxicity: From Mechanisms to Development of Efficient Therapy. IntechOpen: In Cardiotoxicity.

24. Liu, X., Chen, Z., Chua, C. C., Ma, Y. S., Youngberg, G. A., Hamdy, R., et al. (2002). Melatonin as an effective protector against doxorubicin-induced cardiotoxicity. American journal of physiology-heart and circulatory physiology, 283(1), H254-H263.

25. Ammar, E. S. M., Said, S. A., El-Damarawy, S. L., \& Suddek, G. M. (2013). Cardioprotective effect of grape-seed proanthocyanidins on doxorubicin-induced cardiac toxicity in rats. Pharmaceutical biology, 51(3), 339-344.

26. Aygun, H., \& Gul, S. S. (2019). Cardioprotective effect of melatonin and agomelatine on doxorubicin-induced cardiotoxicity in a rat model: an electrocardiographic, scintigraphic and biochemical study. Bratislavske lekarske listy, 120(4), 249-255. 
27. Hazari, M. S., Haykal-Coates, N., Winsett, D. W., Costa, D. L., \& Farraj, A. K. (2009). Continuous electrocardiogram reveals differences in the short-term cardiotoxic response of WistarKyoto and spontaneously hypertensive rats to doxorubicin. Toxicological sciences, 110(1), 224-234.

28. Venugopal, A., Rajasree, O., \& Koshy, R. C. (2014). Effect of anthracyclines and isoflurane on QTc interval. Egyptian Journal of Anaesthesia, 30(1), 83-87.

29. Guo, Z., Yan, M., Chen, L., Fang, P., Li, Z., Wan, Z., et al. (2018). Nrf2-dependent antioxidant response mediated the protective effect of tanshinone IIA on doxorubicin-induced cardiotoxicity. Experimental and therapeutic medicine, 16(4), 3333-3344.

30. Zare, M. F. R., Rakhshan, K., Aboutaleb, N., Nikbakht, F., Naderi, N., Bakhshesh, M., et al. (2019). Apigenin attenuates doxorubicin induced cardiotoxicity via reducing oxidative stress and apoptosis in male rats. Life sciences, 232, 116623.

31. Takemura, G., \& Fujiwara, H. (2007). Doxorubicin-induced cardiomyopathy: from the cardiotoxic mechanisms to management. Progress in cardiovascular diseases, 49(5), 330-352.

32. Meng, Y. Y., Yuan, Y. P., Zhang, X., Kong, C. Y., Song, P., Ma, Z. G., et al. (2019). (2019. Oxidative medicine and cellular longevity: Protection against Doxorubicin-Induced Cytotoxicity by Geniposide Involves AMPK $\alpha$ Signaling Pathway.

33. Attia, S. M., \& Bakheet, S. A. (2013). Effect of dihydrokainate on the capacity of repair of DNA damage and apoptosis induced by doxorubicin. Mutagenesis, 28(3), 257-261.

34. Hassan, A., Ibrahim, A., Mbodji, K., Coeffier, M., Ziegler, F., Bounoure, F., et al. (2010). An $\alpha$-linolenic acid-rich formula reduces oxidative stress and inflammation by regulating NF- $\mathrm{\kappa B}$ in rats with TNBS-induced colitis. The Journal of nutrition, 140(10), 1714-1721.

35. Baghel, S. S., Shrivastava, N., Baghel, R. S., Agrawal, P., \& Rajput, S. (2012). A review of quercetin: antioxidant and anticancer properties. World J Pharm Pharmaceutical Sci, 1(1), 146-160.

36. Nguyen, T., Yang, C. S., \& Pickett, C. B. (2004). The pathways and molecular mechanisms regulating Nrf2 activation in response to chemical stress. Free Radical Biology and Medicine, 37(4), 433-441.

37. Turrens, J. F. (2003). Mitochondrial formation of reactive oxygen species. The Journal of physiology, 552(2), 335-344.

38. De Vries, H. E., Witte, M., Hondius, D., Rozemuller, A. J., Drukarch, B., Hoozemans, J., \& van Horssen, J. (2008). Nrf2-induced antioxidant protection: a promising target to counteract ROSmediated damage in neurodegenerative disease. Free Radical Biology and Medicine, 45(10), 1375-1383.
39. Lin, M. T., \& Beal, M. F. (2006). Mitochondrial dysfunction and oxidative stress in neurodegenerative diseases. Nature, 443(7113), 787-795.

40. Wang, L., Ding, L., Yu, Z., Zhang, T., Ma, S., \& Liu, J. (2016). Intracellular ROS scavenging and antioxidant enzyme regulating capacities of corn gluten meal-derived antioxidant peptides in HepG2 cells. Food Research International, 90, 33-41.

41. Desnoyers, M., Gilbert, K., Madingou, N., Gagné, M. A., Daneault, C., Des Rosiers, C., et al. (2018). A high omega-3 fatty acid diet rapidly changes the lipid composition of cardiac tissue and results in cardioprotection. Canadian journal of physiology and pharmacology, 96(9), 916-921.

42. Papaiahgari, S., Zhang, Q., Kleeberger, S. R., Cho, H. Y., \& Reddy, S. P. (2006). Hyperoxia stimulates an Nrf2-ARE transcriptional response via ROS-EGFR-PI3K-Akt/ERK MAP kinase signaling in pulmonary epithelial cells. Antioxidants \& redox signaling, 8(1-2), 43-52.

43 Barakat, B. M., Ahmed, H. I., Bahr, H. I., \& Elbahaie, A. M. (2018). Protective effect of boswellic acids against doxorubicininduced hepatotoxicity: impact on Nrf2/HO-1 defense pathway. Oxidative medicine and cellular longevity. https://doi.org/10. 1155/2018/8296451

44. Zeng, Y. Y., Jiang, W. D., Liu, Y., Wu, P., Zhao, J., Jiang, J., et al. (2016). Dietary alpha-linolenic acid/linoleic acid ratios modulate intestinal immunity, tight junctions, antioxidant status and mRNA levels of NF-kB p65, MLCK and Nrf2 in juvenile grass carp (Ctenopharyngodonidella). Fish \& shellfish immunology, 51, 351-364.

45 Eraky, S. M., \& El-Magd, N. F. A. (2020). Omega-3 fatty acids protect against acetaminophen-induced hepatic and renal toxicity in rats through HO-1-Nrf2-BACH1 pathway. Archives of Biochemistry and Biophysics. https://doi.org/10.1016/j.abb.2020. 108387

46. Hajra, S., Basu, A., Singha Roy, S., Patra, A. R., \& Bhattacharya, S. (2017). Attenuation of doxorubicin-induced cardiotoxicity and genotoxicity by an indole-based natural compound 3, 3'-diindolylmethane (DIM) through activation of Nrf2/ARE signaling pathways and inhibiting apoptosis. Free Radical Research, 51(9-10), 812-827.

Publisher's Note Springer Nature remains neutral with regard to jurisdictional claims in published maps and institutional affiliations. 